\title{
Über Jubiläen und ihre Fallstricke
}

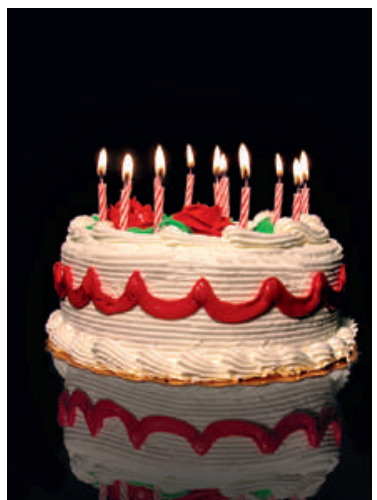

* Die Literatur und weitere Angaben finden sich im Internet unter www.saez.ch $\rightarrow$ aktuelle Ausgabe oder $\rightarrow$ Archiv $2012 \rightarrow 48$

** PD Dr. rer. soc. Eberhard Wolff ist Kulturwissenschaft ler, Medizinhistoriker und Mitglied der Redaktion Geschichte der Schweizerischen Ärztezeitung.
Runde Geburtstage sind eine famose Sache. Wenn Onkel Oskar 60 wird, lädt er die ganze Verwandtschaft zu einem feinen Znacht ein, und jemand hält eine Lobrede. Auch in den Medien lässt sich ein «Vor genau einhundert Jahren ...» einfach verkaufen. Davon habe ich übrigens schon öfters dankbar profitiert.

Neulich machte mich ein Kollege auf den jährlich erscheinenden Bildkalender «Zur Geschichte der Medizin» im Vitalis-Verlag [1] aufmerksam. Er bietet jede Woche ein mehr oder weniger rundes Jubiläum. Daraus entsteht ein ziemlich bunter Cocktail aus Geschichten. Sie spannen sich von Paracelsus (vor 520 Jahren geboren) bis zur Doppelhelix (vor 60 Jahren erstmals als Hypothese). Alles gewürzt mit Illustrationen, Zitaten, einem Erläuterungstext.

Das Rezept dieser Attraktivität ist einfach. Man nehme mehr oder weniger bekannte Entdeckungen und Entdecker wie Emile Roux (starb vor 80 Jahren) oder Robert Koch (vor 170 Jahren geboren) und mische sie mit Ärzten, die es sonst zu Bekanntheit gebracht haben, wie Jean-Paul Marat (vor 270 Jahren geboren). So weit, so gut.

Doch hat die Methode «Erfolg und Berühmtheit» ihre Fallstricke. Bei dieser Glanz-und-GloriaMedizingeschichte (übrigens ausschliesslich männlichen Geschlechts) wird einem ganz schummerig vor lauter «wegweisend», «hervorragend» und «sensationell», vor lauter «Pionier», «Koryphäe» und «Titan».

Wenn die Geburtstagsrede Onkel Oskar über den grünen Klee lobt, staunen die Uneingeweihten. Die anderen kennen die Umwege und dunkleren Seiten seines Lebens. Brüche und Widersprüche werden einfach weggejubelt. Etwa dass Robert Koch mit seinem Tuberkulose-Impfstoff «Tuberkulin» grandios scheiterte. Manchmal wird dies auch dem Kalenderautor klar: Über Franz Joseph Galls «berühmte» Schädellehre heisst es einige Zeilen später beiläufig, dass sie sich «nicht lange halten» konnte.

Wer in der Geschichte der Medizin nur den «Ehrenplatz» sucht, verbaut sich die Chance, Geschichte tiefer zu verstehen. Dass die Heiligen Cosmas und Damian eben nicht, wie immer wieder kolportiert, die «erste erfolgreiche Organtransplantation» vollbracht haben [2]. Oder dass Antony van Leeuwenhoek mit seinem frühen Mikroskopieren im 17. Jahrhundert eben nicht Bakterien in unserem heutigen Verständnis als Infektionsherde entdeckt hat.

Die vordergründige Sicht führt schnell auf ein abschüssiges Gleis. Ein Beispiel: Der deutsche Psychiater Ernst Kretschmer (125 Jahre alt) habe sich mit einer «biologisch fundierten Konstitutionstypologie» «einen Namen gemacht». Er unterschied ab den 1920er Jahren «muskulöse «Athleten`, magere «Leptosome», rundliche «Pykniker» und missgebildete «Dysplastiker . Jedem dieser Typen wies er charakterliche Grundeigenheiten und, als Besonderheit, auch jeweils bestimmte Dispositionen zu psychischen Erkrankungen zu.» Solche «Studien über den Zusammenhang zwischen der körperlichen und der geistigen Verfassung» seien «zu ihrer Zeit einflussreich» gewesen.

Genau. Doch hier, wo es interessant wird, bricht der Kalender ab. Dabei ist die damals verbreitete Verbindung von körperlichen mit psychischen Eigenschaften eine überaus delikate Angelegenheit. Sie diente der pathologisierenden Ausgrenzung des Andersseins etwa über die Physiognomie als «Neuropathen» [3]. Der «wissenschaftliche» Psychiaterblick war obendrein durch zeitgenössische Schönheitsund Anstandsnormen gefärbt [4].

In Verbindung mit erbbiologischen Ideen und den Degenerationsfantasien der Zeit erwuchs daraus die Forderung nach «Unfruchtbarmachung». Auch für Kretschmer: Im NS-Zwangssterilisierungsgesetz sah er einen Weg, seine Ideen «züchterisch verwerten» zu können, und «sehr wichtige Möglichkeiten» für die «Ausmerzung krankhafter Erbanlagen», etwa bei Sippen, die «biologisch minderwertig und entartet» sind [5]. Nichts davon lässt uns der Kalender wissen. Ist das zu viel Hintergrund? Oder steckt hier noch mehr dahinter? Präsentiert der Kalender doch den NS-Anatomen Eduard Pernkopf als Opfer [6].

Es greift jedoch zu kurz, nur den NS-Detektor einzuschalten und «Skandal» Zu deklamieren. Medizingeschichte muss Zusammenhänge herstellen, damit wir die Geschichte besser verstehen. Und manchmal muss sie auch Abgründe ausloten.

Auf dem 41. Blatt des besagten Kalenders prangt das Kretschmer-Zitat: «Wissenschaft ist eine Frage des Charakters, der strengen Zucht und des Verzichts, eine Frage der Redlichkeit, der Unerbittlichkeit, der aufrechten Gesinnung und eines unendlichen Leistungswillens.» Für sich gelesen klingt das Zitat bestenfalls nach pathetischer Selbststilisierung. Im grösseren Zusammenhang lassen mich die markigen Worte erschauern. Hier müsste man systematisch weiterdenken.

Ein runder Geburtstag ist eine famose Sache. Medizingeschichte auch, aber sie hat so viele Fallstricke, dass man vorsichtig sein sollte, wenn man sie auf dem Niveau einer Geburtstagsfeier abhandelt.

Eberhard Wolff** 
1 Salfellner T. Zur Geschichte der Medizin. Aus dem Weltreich der Heilkunst und Pharmazie. 2013. Prag: Vitalis-Verlag; 2012.

2 Schlich T. How Gods and Saints Became Transplant Surgeons: the Scientific Article as a Model for the Writing of History. History of Science 1995;33: 311331.

3 Neu z. B. Ritzmann I. Lesarten des Körpers im Zeitalter der Eugenik. In: Baer J, Rother W (eds.). Körper: Aspekte der Körperlichkeit in Medizin und Kulturwissenschaften. Basel: Schwabe; 2011; p. 43—63.

4 Regener S. Visuelle Gewalt. Menschenbilder aus der Psychiatrie des 20. Jahrhunderts. Bielefeld: Transcript; 2010.

5 Kretschmer E. Konstitutionslehre und Rassenhygiene. In: Rüdin E (ed.): Erblehre und Rassenhygiene im völkischen Staat. München: Lehmanns; 1934, p. 184-93, hier 184-5. Weitere Literaturverweise zu Kretschmers Verbindungen mit dem Nationalsozialismus z.B. in seinem Wikipedia-Eintrag.

6 Der österreichische Anatom und Herausgeber des bekannten Anatomieatlasses Eduard Pernkopf war bekennender Nationalsozialist (1933 NSDAP-Mitglied, 1943 Rektor der Universität Wien, SA-Sturmbannführer). Die Präparate, die als Vorlagen seiner Abbildungen dienten, können nicht einzeln und zweifelsfrei als Opfer des Unrechtsregimes identifiziert werden, aber die Indizien machen es sehr wahrscheinlich. Der Kalendereintrag stellt Pernkopf als «Verfemten» und Opfer der NS-Aufarbeitung dar: «Über Pernkopfs exponierte Stellung ist Gras gewachsen, als die Enkelgeneration erneut über den mehr als ein halbes Jahrhundert Toten zu Gericht sitzt und ihn und sein Werk der ewigen Verdammnis anheimstellt. Seine wissenschaftlichen Leistungen werden fortan unter Anführungszeichen gesetzt. Die Inquisition hat gesprochen, Vesalius verhülle sein Haupt.» $\mathrm{Zu}$ Pernkopf siehe Hubbard C. Eduard Pernkopf's Atlas of Topographical and Applied Human Anatomy: The Continuing Ethical Controversy. The Anatomical Record 2001;265:20711. Lenzen-Schulte M:

Die Anatomen der braunen Henker. Frankfurter Allgemeine Zeitung, 14. 7. 2010. 